\title{
Measurement properties of self-report pedestrians' road crossing behavior questionnaires constructed based on the theory of planned behavior: protocol for a systematic review
}

Mahdi Moshki ${ }^{1}$, Abdoljavad Khajavi ${ }^{2}$, Fatemeh Sadeghi-Ghyassi ${ }^{3}$, Homayoun Sadeghi-Bazargani ${ }^{4}$ and Saeid Pour-Doulati, ${ }^{5,6^{*}}$ (D)

\begin{abstract}
Background: Pedestrians' unsafe crossing behavior exposes them at risk of trauma and death and puts a tremendous burden on the health care system. The theory of planned behavior (TPB) is one of the leading theoretical models used to develop pedestrians' road crossing behavior questionnaires, yet the quality of measurement properties of them has not been evaluated. The aim of the proposed systematic review is to evaluate the quality of measurement properties of the questionnaires constructed based on the TPB to predict pedestrians' road crossing behavior.

Methods: We will include studies validating or evaluating one or more psychometric properties of the self-reported questionnaire employing the TPB for predicting pedestrians' road crossing behavior. A comprehensive search strategy will be formulated based on the components of review aim. The databases of MEDLINE, Embase, PubMed, Cochrane Library, PsycINFO, PsycARTICLES, and ProQuest, also grey literature and the reference lists of the included studies, will be searched. A hand search for the relevant journals and Google Scholar will be conducted. COnsensus-based Standards for the selection of health Measurement INstruments (COSMIN) Risk of Bias checklist will be used to evaluate the measurement properties of the included questionnaires. First, we will assess standards for the methodological quality of each study. Then, each scale or subscale of a questionnaire will be rated using the updated criteria for good measurement property. We will quantitatively pool or qualitatively summarize the results and will evaluate them against the criteria for good measurement properties. Finally, we will grade the pooled or summarized evidence using the GRADE (Grading of Recommendations, Assessment, Development, and Evaluation) approach and provide recommendations for the most appropriate instrument.
\end{abstract}

Discussion: The proposed systematic review will evaluate the measurement properties of self-report pedestrians' road crossing behavior questionnaires constructed based on the TPB. The findings will help researchers in selecting the appropriate TPB-based instrument for pedestrians' road crossing behavior.

Systematic review registration: PROSPERO CRD42017047793

Keywords: COSMIN, Pedestrian, Questionnaire, Theory of planned behavior, Quality assessment

\footnotetext{
* Correspondence: s.pourdoulati@gmail.com

${ }^{5}$ Social Development \& Health Promotion Research Center, Gonabad

University of Medical Sciences, Gonabad, Iran

${ }^{6}$ Center for Non-Communicable Diseases Control and Prevention, East

Azerbaijan Province Health Center, Tabriz 5143814998, Iran

Full list of author information is available at the end of the article
}

(c) The Author(s). 2019 Open Access This article is distributed under the terms of the Creative Commons Attribution 4.0 International License (http://creativecommons.org/licenses/by/4.0/), which permits unrestricted use, distribution, and reproduction in any medium, provided you give appropriate credit to the original author(s) and the source, provide a link to the Creative Commons license, and indicate if changes were made. The Creative Commons Public Domain Dedication waiver (http://creativecommons.org/publicdomain/zero/1.0/) applies to the data made available in this article, unless otherwise stated. 


\section{Background Rationale}

Pedestrian injuries and deaths should be considered as a growing public health problem [1-3]. Pedestrians are amongst the most vulnerable road users globally, accounting for $23 \%$ of the world's road accident deaths in 2018 [4]. Unsafe road crossing behavior exposes them to risk of trauma and death and imposes a heavy burden on the health care system $[5,6]$. The United Nations (UN) recommends governments to pay more attention to vulnerable road users such as pedestrians, by making proper policies and practices for pedestrian safety [7]. The high rate of pedestrian injury and mortality highlights the importance of an urgent call for evidencebased health education and safety promotion programs [8]. Nevertheless, research and interventions to improve pedestrian safety are still unsatisfactory $[4,9,10]$.

Ensuring appropriate targets for intervention [9], the theory-driven interventions are more effective than interventions lacking theory [10-12]. The theory of planned behavior (TPB) developed by Ajzen [13] is one of the most frequently applied social-psychological models and helps health professionals to identify key beliefs to develop appropriate health behavior change intervention [11, 14, 15]. A number of researchers have used the TPB to predict pedestrians' intention to cross the road in a potentially hazardous situation [16-24]. The TPB renders an authoritative framework for conceptualization, measurement, and identifying issues affecting behavior [11]. The TPB argues that behavioral intention and perceived behavioral control (PBC) are proximal predictors of actual behavior and is determined by its three basic constructs, including attitude toward the behavior, subjective norms, and $\mathrm{PBC}[14,22]$. In order to measure intention, it is necessary to measure its predictors. These predictors are latent variables and should be measured indirectly through a questionnaire response [14].

Development of a valid and reliable questionnaire as a measurement instrument is a very critical point, particularly in social-psychological and health-related behavior research. Due to some confusing issue and diverse views about the operationalization of the TPB, particularly for non-psychologist researchers[14], Ajzen [25], Francis et al. [26], Fishbein and Ajzen [27], Conner and Norman [28], and Godin and Kok [29] proposed some sort of guidelines for constructing the TPB-based questionnaire to help the researchers in developing a valid and reliable tool. In developing a TPB-based questionnaire, initial qualitative study, the wording of questions, response formats, and scoring should be considered as essential steps [14]. Different questionnaires based on the TPB have been used to measure pedestrians' road crossing intention/ behavior worldwide [16-24, 30]. To what extent these questionnaires enjoy satisfactory measurement properties (MPs) is unknown. COSMIN (COnsensus-based Standards for the selection of health Measurement Instruments) initiative, is an international multidisciplinary team of researchers which has provided a new updated modular tool for conducting a systematic review of patient-reported outcome measures (PROMs) and evaluating the outcome measurement instruments [31]. COSMIN methodology uses the word patient but, the target population could be not the patient. In this study, the target population is pedestrian, and the outcome measure is the pedestrians' road crossing behavior. So, here the capital letter $\mathrm{P}$ in the PROMs represents pedestrian instead of patient or in general let us say, participant. Systematic reviews of PROMs provide an overall understanding of the MPs of PROMs to select the most suitable PROM for a certain objective [31]. An initial search conducted within the Cochrane Database of Systematic Reviews, PubMed $\backslash$ MEDLINE, and PROSPERO indicates that the only systematic review for evaluating the quality of TPB-based questionnaires and their development processes has been conducted by Oluka et al. in 2014, regardless of concentration on a specific subject like pedestrian behavior [32]. Since there are no current or underway systematic reviews on the topic, a systematic review is needed and reasonable to identify, appraise, summarize, and compare the quality of MPs of available self-reported TPB-based questionnaires predicting pedestrians' road crossing intention or behavior.

So the aim of the proposed systematic review is to evaluate the quality of MPs of the questionnaires constructed based on the theory of planned behavior (TPB) to predict pedestrians' road crossing intention/behavior by using the updated COSMIN methodology. The following specific objectives will be achieved by the proposed systematic review.

1. To identify the existing TPB-based questionnaires predicting pedestrians' road crossing intention or behavior

2. To evaluate the methodological quality of the included studies

3. To assess the quality of MPs

4. To grade the overall quality of evidence

5. To provide recommendation on the most suitable questionnaires

\section{Methods}

A multidisciplinary research team which consists of the following members prepared this protocol and will conduct the main review: MM, a full professor in Health Education \& Promotion, is an expert in the theory of planned behavior. SP, a Ph.D. candidate of Health Promotion, concentrated in pedestrian injury prevention and safety promotion. AK, an associate professor of Health Service Management, is an expert in systematic review methodology and measurement instruments. $\mathrm{HS}$ is an associate professor of Epidemiology 
specialist in the field of Statistical Modeling concentrated in Injury Prevention and Safety Promotion. He is also a lecturer of the JBI (Joanna Briggs Institute) Database of Systematic Reviews and Implementation Reports. FS, a Ph.D. candidate of Health Information Management, is a skilled librarian, specialist in systematic search, a member of National Research Center for EvidenceBased Medicine, and also a member of JBI and lecturer of systematic search strategies and systematic review.

This protocol has been registered in the International Prospective Register of Systematic Review (PROSPERO): CRD42017047793. We followed recommendations of the Preferred Reporting Items for Systematic Reviews and Meta-Analyses Protocols (PRISMA-P) statement [33] and the COSMIN for conducting a systematic review of PROMs [34]. The first version of COSMIN [35] only provided "standards" that concern study design and statistical methods used to assess the methodological quality of the MPs. We will use the last version of COSMIN methodology [34] which also developed "criteria" that concerns the quality of PROM and provides gold standards for good MPs. The COSMIN methodology was originally developed to use for the PROMs which are used for evaluative purposes, but it is applicable for predictive application, as suggested by its developers [30]. A PRISMA-P checklist for this protocol is available in Additional file 1.

\section{Inclusion criteria}

The following four key elements of a systematic review of PROMs will be used to formulate eligibility criteria:

\section{Construct}

The construct of the study is pedestrian road crossing behavior. By road crossing behavior, we mean both safe and unsafe road crossing behavior, but not railroad crossing behavior. The first one keeps them safe from collision with road vehicles, and the second one has the potential of collision with road vehicles and subsequently could lead to injury and even death.

\section{Population of interest}

We will consider studies that have included pedestrians of any age.

\section{Type of instrument}

We will consider primary studies that have presented pedestrian self-reported TPB-based questionnaire to predict road crossing behavior or intention.

\section{Measurement properties}

We will consider studies that have reported on MPs concerning the content validity including face validity, structural validity, consistency, cross-cultural validity or measurement invariance, reliability, measurement error, hypothesis testing, and responsiveness.

\section{Inclusion criteria}

We will include original studies that have employed the TPB as a theoretical framework for predicting pedestrians' road crossing intention or behavior, where the study is a validation study or evaluating one or more psychometric properties of the self-reported questionnaire. We will not consider any restrictions on the type of population or participants, geographical location, language, setting, and time. Although we will not restrict the search strategy to the type of language, we will include only articles written in English. The nonEnglish studies will be reported merely to inform readers about the existence of such questionnaires [31].

\section{Exclusion criteria}

The exclusion criteria will be as follows: (1) full-text not available, (2) duplicate publications or sub-studies of included research, and (3) studies that only use self-reported TPB-based questionnaire as an outcome measurement instrument.

\section{Search strategy}

Pertinent keywords obtained from the preliminary search were tested and verified by a qualified reviewer (FS). The agreed keywords based on the main components of the review aim and consistent with the inclusion criteria will be used to formulate the search strategy which consists of a comprehensive set of search terms including index terms and free text words for:

1. Construct of interest: road crossing behavior

2. Population of interest: pedestrians

3. Measurement properties: content validity including face validity, structural validity, consistency, crosscultural validity or measurement invariance, reliability, measurement error, hypothesis testing, and responsiveness

4. Type of instrument: self-reported TPB-based questionnaire

The Ovid MEDLINE ${ }^{\oplus}$ search strategy will be tailored to other databases. Two librarians (FS and FA) will help to formulate search strategy and then do search independently. The PRESS checklist for MEDLINE and Embase databases will be filled up by a medical librarian $(\mathrm{Z} \mathrm{F})$, who is an expert in a systematic review, and at the same time, she does not cooperate with the team. A draft search strategy for Ovid MEDLINE ${ }^{\oplus}$ is provided in Table 1. 
Table 1 Search strategy developed for Ovid MEDLINE ${ }^{\oplus}$

\begin{tabular}{|c|c|}
\hline Set & Strategy \\
\hline 1 & (behavior\$ or behaviour\$).tw. \\
\hline 2 & $\begin{array}{l}\text { (intention\$ or predict\$ or impress\$ } \\
\text { or feeling\$ or attitude\$ or decision\$).tw. }\end{array}$ \\
\hline 3 & (belief or believes).tw. \\
\hline 4 & exp Behavior/ (1670182) \\
\hline 5 & exp Intention/ \\
\hline 6 & exp Attitude/ \\
\hline 7 & 1 or 2 or 3 or 4 or 5 or 6 \\
\hline 8 & cross\$.tw. \\
\hline 9 & 7 or 8 \\
\hline 10 & pedestrian\$.tw. \\
\hline 11 & traffic\$.tw. \\
\hline 12 & exp Accidents, Traffic/ or exp Pedestrians/ \\
\hline 13 & 10 or 11 or 12 \\
\hline 14 & 9 and 13 \\
\hline 15 & exp "Surveys and Questionnaires"/ \\
\hline 16 & $\begin{array}{l}\text { (questionnaire\$ or inventor\$ or scale\$ or } \\
\text { instrument\$ or assessment\$ or measure\$ } \\
\text { or survey\$).tw. }\end{array}$ \\
\hline 17 & 15 or 16 \\
\hline 18 & $\begin{array}{l}\text { (psychometr\$ or valid\$ or reliab\$ or } \\
\text { consistenc\$).tw. }\end{array}$ \\
\hline 19 & (hypothesis adj2 test\$).tw. \\
\hline 20 & (test adj2 retest).tw. \\
\hline 21 & exp Psychometrics/ \\
\hline 22 & exp Validation Studies/ \\
\hline 23 & 18 or 19 or 20 or 21 or 22 \\
\hline 24 & 17 and 23 \\
\hline 25 & 14 and 24 \\
\hline 26 & (reasoned adj3 action adj3 approach\$).tw. \\
\hline 27 & (theory adj5 planned adj3 behavio?r\$).tw. \\
\hline 28 & (theory adj5 reasoned adj3 action).tw. \\
\hline 29 & (TPB or TRA or RAA).tw. \\
\hline 30 & 26 or 27 or 28 or 29 \\
\hline 31 & 25 and 30 \\
\hline 32 & PEROB.tw. \\
\hline 33 & $\begin{array}{l}\text { (Pedestrian\$ adj3 Road\$ adj3 Cross\$ adj3 } \\
\text { Behavio?r\$).af. }\end{array}$ \\
\hline 34 & 32 or $33(13)$ \\
\hline 35 & 31 or $34(20)$ \\
\hline
\end{tabular}

\section{Information sources}

We will conduct an electronic search through the following bibliographic databases: MEDLINE (via Ovid), Embase, PubMed, Cochrane Library, PsycINFO (via EBSCO), PsycARTICLES (via EBSCO), and ProQuest. We will also search grey literature and will check the reference lists of the included studies. The databases of SCOPUS and Web of Science will be used for citation tracking to include the articles which have cited the included articles if they meet the inclusion criteria. Along with electronic search, we will fulfill a hand search for the relevant journals like Transportation Research Part F: Traffic Psychology and Behavior, and Accident analysis; and Google Scholar.

\section{Study records}

\section{Data management}

We will export the results of database searches to EndNote reference management software (VX6) to manage records during the review and remove duplicate references.

\section{Selection process}

Two independent reviewers (SP, MM) will screen the title and then abstracts of the retrieved articles. If they meet the inclusion criteria, reviewers will read the full texts and assess them against the inclusion criteria. They will scan the reference lists of included studies to ensure literature saturation. In the case of any disagreement, the reviewer will discuss for clarification and resolution. If they could not reach a consensus, a third party (AK) will arbitrate. We will calculate inter-rateragreement using Cohen's $\kappa$ to verify the level of agreement between two reviewers. Justification for the excluded articles will be provided. A PRISMA flow diagram will demonstrate included and excluded studies as depicted in Additional file 2 [36].

\section{Assessment of methodological quality}

The COSMIN Risk of Bias (RoB) checklist will be used by two independent reviewers (SP, MM) to evaluate the MPs of the included PROMs. The COSMIN RoB checklist contains ten boxes to assess required standards for good methodological quality of single studies included in systematic reviews in terms of design requirements and statistical methods/analysis [35, 37]. We will first determine which MPs are assessed in each article to complete the corresponding box of COSMIN RoB checklist for that $\mathrm{MP}$. Content validity $(\mathrm{CV})$ is the most important MP because it makes clear if the items of the PROM are relevant, comprehensive, and comprehensible regarding the construct of interest and study population. So, as a general procedure, we will first evaluate PROM development and CV (boxes 1 and 2, respectively) [37]. If the PROM has sufficient $\mathrm{CV}$, in the second step, we will assess the internal structure of the PROMs, including first, structural validity, followed by internal consistency, and cross-cultural validity or measurement invariance (boxes 3 , 4, and 5 respectively) [37]. Otherwise, we will exclude it from the review. This step will be done if the PROM is based on a reflective model (i.e., a scale or subscale 
manifests itself in the items of one underlying construct). Two types of measures can be applied in the TPB model to predict intention and direct and indirect measures. The indirect measures are formative and the direct measures are reflective indicators of attitude, subjective norm, and perceived behavioral control respectively. Some researchers use only direct measures and some use both. Lastly, we will evaluate the remaining MPs including reliability, measurement error, hypotheses testing for construct validity, and responsiveness (boxes 6-7 and 9-10 respectively) [37]. Criterion validity (box 8) [37] will not be assessed because there is no gold standard for predicting pedestrians' road crossing behavior. As such, we will not use part a of box 10 for assessing responsiveness which is based on criterion approach. We will use parts b, c, and d of the box 10 for assessing responsiveness which based on the construct approach assesses hypotheses testing by comparison with other outcome measurement instruments, between subgroups, and before and after the intervention if it is the case [34].

For the evaluation of each MP of the TPB-based pedestrian behavior, first, we will assess standards for the methodological quality of each study on MP using the corresponding COSMIN RoB checklist and applying a 4-point rating scale: very good, adequate, doubtful, and inadequate. The worst score count principal (i.e., taking the lowest score) will be employed to achieve the overall rating of the methodological quality of every single study on MP. Then, each scale or subscale of a PROM will be rated using the updated (gold) criteria for good MP as sufficient (+), insufficient (-), or indeterminate (?) [34] (Fig. 1).

\section{Data extraction}

We will extract the data on characteristics of the $\operatorname{PROM}(\mathrm{s})$, on characteristics of the included sample(s), on results of the MPs, and on interpretability and feasibility. This information will be presented in overview tables [34]. The characteristics of the included PROMs will consist of the name of the PROMs, reference to the PROM development article, constructs being measured, language and study population for which the PROM was developed, intended context of use, available language version of the PROM, number of scales or subscales, number of items, response options, and recall period. The characteristics of the populations will consist of the geographical location, language, target population, sample size, age, gender, and setting [31]. We will contact the authors of included studies if we need any further information for clarification or obtaining missing data.

\section{Data synthesis}

Using information taken by data extraction tables, if the results of different studies for a given MP are consistent, we will quantitatively pool, otherwise, qualitatively summarize them. The pooled or summarized results of the MP will be

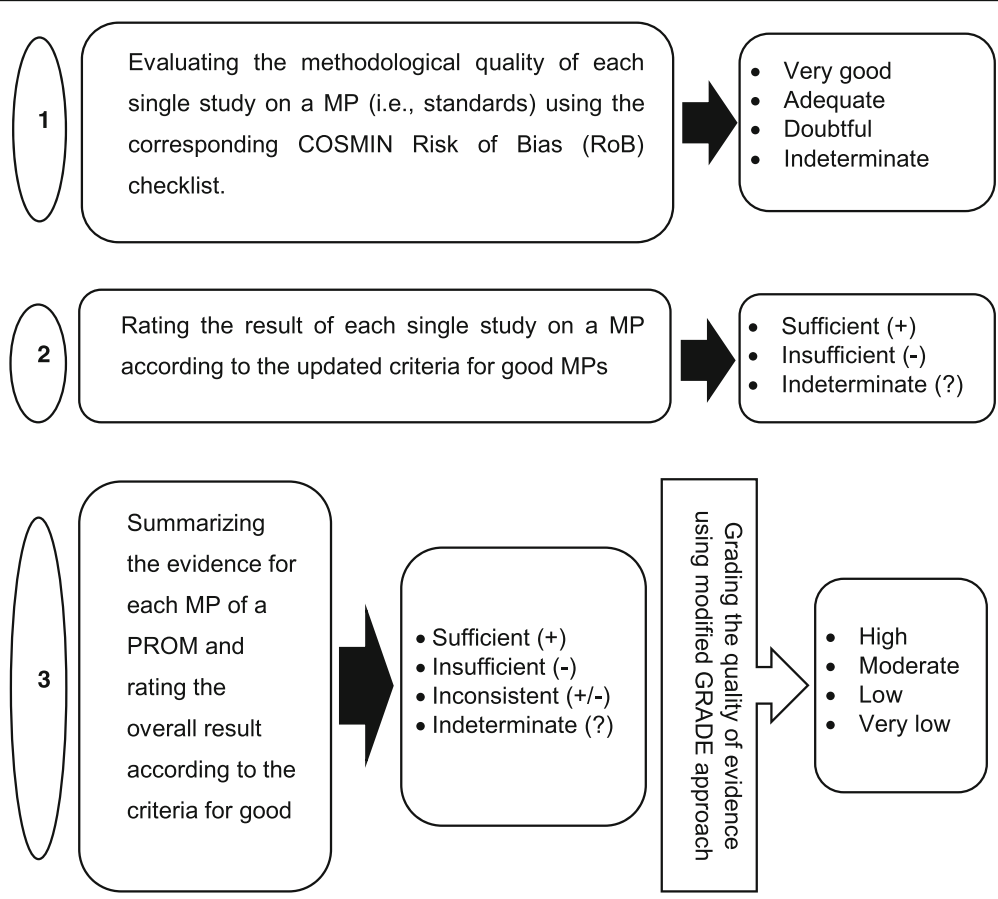

Fig. 1 Evaluation process of each MP. Adapted from "COSMIN methodology for systematic reviews of Patient-Reported Outcome Measures (PROMs) user manual Version 1.0 dated February 2018", by Mokkink LB, Prinsen C, Patrick DL, Alonso J, Bouter LM, de Vet HC, et al., (2018, February). Retrieved from https://www.cosmin.nl/ 
reported in Summary of Finding (SoF) tables [34] and will again be evaluated against the gold criteria for good MPs to get an overall rating as sufficient $(+)$, insufficient $(-)$, inconsistent $( \pm)$, or indeterminate (?). Unlike the evaluation of the MP studies, the focus here is on the PROM itself, not on the single study. To be confident how the overall ratings are trustworthy, we will apply a modified GRADE (Grading of Recommendations, Assessment, Development, and Evaluation) approach for grading the pooled or summarized evidence as high, moderate, low, or very low (Fig. 1). Using the modified GRADE approach, we will consider four factors: risk of bias, inconsistency, imprecision, and indirectness to downgrade the quality of evidence ranging from one to three levels when the trustworthiness of the results is problematic [27]. Risk of bias refers to the methodological quality of the studies, inconsistency concerns the unexplained discrepancy of the results between studies, imprecision refers to low sample size, and indirectness means that the populations, interventions, or outcomes of the evidence differ from what the review interested in [37]. Imprecision, due to not being a matter of concern for $\mathrm{CV}$, will not be considered for grading the quality of $\mathrm{CV}$. COSMIN RoB checklists and detailed instructions on rating each standard are described elsewhere [38].

\section{Developing recommendations to use}

Finally, we will classify the included questionnaires into one of the three groups:

Group "A" recommended for use and results of them would be trusted (i.e., questionnaires with sufficient content validity evidence and at least low evidence for sufficient internal consistency). Group "B" has the potential to recommend, but additional research is needed to reassess the quality of them (i.e., the questionnaires classified not in group "A" or group "C"). Group "C" will not recommend for use because there is strong evidence for an insufficient MP [31].

\section{Discussion}

The proposed systematic review will identify and evaluate self-report questionnaires that expected to predict pedestrians' road crossing behavior. Such a review has not been done before. The findings will reveal the existing self-report pedestrians' road crossing behavior questionnaires constructed based on the TPB and will show the overall quality of evidence and provide recommendation for researchers to identify and select the most appropriate instrument to predict pedestrians' road crossing intention/behavior and to determine their underlying beliefs of attitude, subjective norms, and perceived behavioral control.

\section{Additional files}

Additional file 1: PRISMA-P 2015 checklist. (DOCX 26 kb)

Additional file 2: PRISMA flow diagram example. (DOCX 29 kb)

\section{Abbreviations}

COSMIN: COnsensus-based Standards for the selection of health Measurement Instruments; EBSCO: Elton B. Stephens Co; Embase: Excerpta Medica database; GRADE: Grading of Recommendations, Assessment, Development, and Evaluation; MEDLINE: Medical Literature Analysis and Retrieval System Online; MP: Measurement property; PBC: Perceived behavioral control; PROSPERO: International Prospective Register of Systematic Reviews; PRISMA-P: Preferred Reporting Items for Systematic Reviews and Meta-Analyses Protocols; PROM: Patient-reported outcome measures; PsychINFO: Psychological Information Database; RoB: Risk of bias; SoF: Summary of findings; TPB: Theory of planned behavior; UN: United Nations

\section{Acknowledgements}

We thank Dr. Caroline B Terwee, the founder of the COSMIN initiative, for her assistance in reviewing the manuscript and making valuable comments on it.

Authors' contributions

SP conceptualized the research idea, initiated the protocol, and wrote the manuscript. AK and MM contributed to the design of protocol and provided critical revision of the manuscript. SP and MM drafted the inclusion and exclusion criteria and provided the data extraction forms and training manual. FSG helped to formulate the search strategy and applied it for MEDLINE database. AK helped to write synthesis section and provided methodological advice. HSB reviewed the whole manuscript and provided substantial comments for editing. All authors critically reviewed the study protocol and approved the final manuscript.

\section{Authors' information}

SP is a registered PhD student of Health Promotion at the Gonabad University of Medical Sciences. MM is the full professor at the Gonabad University of Medical Sciences, and he is the overall supervisor of the doctoral thesis. AK is an associate professor of Health Service Management at the Gonabad University of Medical Sciences and performing as a co-supervisor. HSB is associate professor of epidemiology at the Tabriz University of Medical Sciences and performing as a technical advisor. FSG is a PhD candidate of Health Information Management, and she is a senior information specialist working at the Research Center for Evidence Based Medicine, Tabriz University of Medical Sciences.

\section{Funding}

This study protocol, and subsequent systematic review, is a part of a Ph.D. project. Funding is provided by the Social Development \& Health Promotion Research Center, Gonabad University of Medical Sciences, Gonabad, Iran. The funder had no involvement in the study design, writing the manuscript, or submitting for publication.

\section{Availability of data and materials}

Not applicable (this is a systematic review protocol so there are no primary data available).

\section{Ethics approval and consent to participate}

Not applicable

\section{Consent for publication}

Not applicable

\section{Competing interests}

The authors declare that they have no competing interests

\section{Author details}

${ }^{1}$ Health Education and Health Promotion Department, School of Health; Social Development \& Health Promotion Research Center, Gonabad University of Medical Sciences, Gonabad, Iran. ${ }^{2}$ Community Medicine Department, School of Medicine, Gonabad University of Medical Sciences, 
Gonabad, Iran. ${ }^{3}$ Research Center for Evidence Based Medicine, Tabriz University of Medical Sciences, Tabriz, Iran. ${ }^{4}$ Road Traffic Injury Research Center, Tabriz University of Medical Sciences, Tabriz, Iran. ${ }^{5}$ Social Development \& Health Promotion Research Center, Gonabad University of Medical Sciences, Gonabad, Iran. ${ }^{6}$ Center for Non-Communicable Diseases Control and Prevention, East Azerbaijan Province Health Center, Tabriz 5143814998, Iran.

\section{Received: 25 April 2019 Accepted: 26 July 2019}

Published online: 03 August 2019

\section{References}

1. Stoker P, Adkins A, Ewing R. Pedestrian safety and public health. In: Walking. edn. p. 211-29.

2. Chakravarthy B, Lotfipour S, Vaca FE. Pedestrian injuries: emergency care considerations. Cal J Emerg Med. 2007:8(1):15.

3. Organization WH: Pedestrian safety: a road safety manual for decisionmakers and practitioners. 2013

4. WHO: Global status report on road safety 2018. 2018

5. $\quad$ Ding T, Wang S, Xi J, Zheng L, Wang Q. Psychology-based research on unsafe behavior by pedestrians when crossing the street. Adv Mech Eng. 2015;7(1):203867

6. Peden M, Scurfield R, Sleet D, Mohan D, Hyder AA, Jarawan E, Mathers CD. World report on road traffic injury prevention. In: World Health Organization Geneva; 2004

7. United Nations: Improving global road safety, resolution adopted by the General Assembly on 15 April 2016. 2016.

8. Sminkey ML. Global Plan for the Decade of Action for Road Safety 2011 2020. In: World Health Organization www who int/roadsafety/decade_of_ action; 2011.

9. Michie S, Prestwich A. Are interventions theory-based? Development of a theory coding scheme. Health Psychol. 2010;29(1):1.

10. Webb T, Joseph J, Yardley L, Michie S. Using the internet to promote health behavior change: a systematic review and meta-analysis of the impact of theoretical basis, use of behavior change techniques, and mode of delivery on efficacy. J Med Internet Res. 2010;12(1):e4.

11. Glanz K, Rimer BK, Viswanath K: Health behavior and health education: theory, research, and practice, 4th edn: John Wiley \& Sons; 2008.

12. Noar SM, Benac CN, Harris MS. Does tailoring matter? Meta-analytic review of tailored print health behavior change interventions. Psychol Bull. 2007; 133(4):673.

13. Ajzen I. The theory of planned behavior. Organ behav Hum Decis Process. 1991;50(2):179-211.

14. Francis JJ, Johnston M, Eccles MP, Grimshaw J, Kaner EF. Measurement issues in the theory of planned behaviour: a supplement to the manual for constructing questionnaires based on the theory of planned behaviour. Newcastle: Centre for Health Services Research; 2004

15. Epton T, Norman P, Harris P, Webb T, Snowsill FA, Sheeran P: Development of theory-based health messages: three-phase programme of formative research. Health promotion international 2014:dau005.

16. Diaz EM. Theory of planned behavior and pedestrians' intentions to violate traffic regulations. Transport Res Part F: Traffic Psychol Behav. 2002;5(3):169-75

17. Evans D, Norman P. Understanding pedestrians' road crossing decisions: an application of the theory of planned behaviour. Health Educ Res. 1998;13(4):481-9.

18. Evans D, Norman P. Predicting adolescent pedestrians' road-crossing intentions: an application and extension of the Theory of Planned Behaviour. Health Educ Res. 2003:18(3):267-77.

19. Holland C, Hill R. The effect of age, gender and driver status on pedestrians' intentions to cross the road in risky situations. Accid Anal Prev. 2007;39(2):224-37

20. Holland C, Hill R. Gender differences in factors predicting unsafe crossing decisions in adult pedestrians across the lifespan: a simulation study. Accid Anal Prev. 2010;42(4):1097-106.

21. Zhou H, Romero SB, Qin X. An extension of the theory of planned behavior to predict pedestrians' violating crossing behavior using structural equation modeling. Accid Anal Prev. 2015.

22. Zhou R, Horrey WJ. Predicting adolescent pedestrians' behavioral intentions to follow the masses in risky crossing situations. Transportation research part F: traffic psychology and behaviour. 2010;13(3):153-63.
23. Zhou R, Horrey WJ, Yu R. The effect of conformity tendency on pedestrians' road-crossing intentions in China: an application of the theory of planned behavior. Accid Anal Prev. 2009;41(3):491-7.

24. Wang L, Gou J, Zou Q. The Analysis of Pedestrian Violations Based on the Revised TPB (Theory of Planned Behavior). In: LISS 2013. edn.: Springer; 2015 1181-1186.

25. Ajzen I. Constructing a TPB questionnaire: Conceptual and methodological considerations; 2002

26. Francis JJ, Eccles MP, Johnston M, Walker A, Grimshaw J, Foy R, Kaner EF, Smith L, Bonetti D. Constructing questionnaires based on the theory of planned behaviour. In: A manual for health services researchers; 2004.

27. Fishbein $\mathrm{M}$, Ajzen I. Predicting and changing behavior: the reasoned action approach: Taylor \& Francis; 2011.

28. Conner M, Norman P. Predicting health behaviour: McGraw-Hill Education (UK; 2005.

29. Godin G, Kok G. The theory of planned behavior: a review of its applications to health-related behaviors. Am J Health Promot. 1996;11(2):87-98.

30. Jalilian M, Mostafavi F, Mahaki B, Delpisheh A, Rad GS. An application of a theory of planned behaviour to determine the association between behavioural intentions and safe road-crossing in college students: perspective from Isfahan, Iran. JPMA. 2015;65(7):742-6.

31. Prinsen CA, Mokkink LB, Bouter LM, Alonso J, Patrick DL, De Vet HC, Terwee CB. COSMIN guideline for systematic reviews of patient-reported outcome measures, Qual Life Res. 2018:27(5):1147-57.

32. Oluka OC, Nie S, Sun Y. Quality assessment of TPB-based questionnaires: a systematic review. PloS One. 2014;9(4):e94419.

33. Moher D, Shamseer L, Clarke M, Ghersi D, Liberati A, Petticrew M, Shekelle P, Stewart LA. Preferred reporting items for systematic review and metaanalysis protocols (PRISMA-P) 2015 statement. Syst Rev. 2015;4:1):1.

34. Mokkink LB, Prinsen C, Patrick DL, Alonso J, Bouter LM, de Vet HC, Terwee $\mathrm{CB}$, Mokkink L. COSMIN methodology for systematic reviews of patientreported outcome measures (PROMs). User Manual. 2018;78:1.

35. Mokkink LB, Terwee CB, Patrick DL, Alonso J, Stratford PW, Knol DL, Bouter LM, de Vet HC. COSMIN checklist manual. Amsterdam: University Medical Center; 2012.

36. Moher D, Liberati A, Tetzlaff J, Altman DG. Preferred reporting items for systematic reviews and meta-analyses: the PRISMA statement. Ann Intern Med. 2009;151(4):264-9.

37. Mokkink LB, De Vet HC, Prinsen CA, Patrick DL, Alonso J, Bouter LM, Terwee CB. COSMIN risk of bias checklist for systematic reviews of patient-reported outcome measures. Qua Life Res. 2018;27(5):1171-9.

38. Terwee CB, Prinsen C, Chiarotto A, de Vet H, Bouter LM, Alonso J, Westerman MJ, Patrick DL, Mokkink LB: COSMIN methodology for assessing the content validity of PROMs-user manual. 2018.

\section{Publisher's Note}

Springer Nature remains neutral with regard to jurisdictional claims in published maps and institutional affiliations.
Ready to submit your research? Choose BMC and benefit from:

- fast, convenient online submission

- thorough peer review by experienced researchers in your field

- rapid publication on acceptance

- support for research data, including large and complex data types

- gold Open Access which fosters wider collaboration and increased citations

- maximum visibility for your research: over $100 \mathrm{M}$ website views per year

At BMC, research is always in progress.

Learn more biomedcentral.com/submissions 